Original Article - Clinical Science

\title{
Prevalence of trachomatous trichiasis in Australia: the National Eye Health Survey
}

Mohamed Dirani PhD, ${ }^{1}$ Stuart Keel PhD, ${ }^{1}$ J oshua Foreman BSci(Hons) ${ }^{1,2}$ Peter van Wijngaarden FRANZCO ${ }^{1,2}$ and Hugh R Taylor $\mathrm{AC}^{3}$

${ }^{1}$ Centre for Eye Research Australia, Royal Victorian Eye \& Ear Hospital, Melbourne, Australia, ${ }^{2}$ Ophthalmology, University of Melbourne, Department of Surgery, Melbourne, Australia,

${ }^{3}$ Indigenous Eye Health Unit, Melbourne School of Population and Global Health, the University of Melbourne, Melbourne, Australia

${ }^{1}$ Centre for Eye Research Australia, Royal Victorian Eye \& Ear Hospital, Melbourne, Australia

${ }^{2}$ Ophthalmology, University of Melbourne, Department of Surgery, Melbourne, Australia ${ }^{3}$ Indigenous Eye Health Unit, Melbourne School of Population and Global Health, the University of Melbourne, Melbourne, Australia

Correspondence: Dr Mohamed Dirani, Address: Level one, Centre for Eye Research Australia, 32 Gisborne Street, East Melbourne, Victoria, Australia 3002

Email: dirani@unimelb.edu.au

Short running title: Trachoma in Australia

Received 15 February 2017; accepted 1 J une 2017

Conflict of interest: None

This is the author manuscript accepted for publication and has undergone full peer review but has not been through the copyediting, typesetting, pagination and proofreading process, which may lead to differences between this version and the Version of Record. Please cite this article as doi: $10.1111 /$ ceo.13003

This article is protected by copyright. All rights reserved. 
Funding sources: Department of Health of the Australian Government, Peggy and Leslie Cranbourne Foundation and Novartis Australia. In-kind support from our industry and sector partners, OPSM, Carl Zeiss, Designs for Vision, the Royal Flying Doctor Service, Optometry Australia and the Brien Holden Vision Institute. The Centre for Eye Research Australia receives Operational Infrastructure Support from the Victorian Government. The Principal Investigator, Dr Mohamed Dirani, is supported by a NHMRC Career Development Fellowship (\#1090466). The PhD student, J oshua Foreman is supported by an Australian Postgraduate Award scholarship.

\section{ABSTRACT}

I mportance: Australia is the only developed country to still have pockets of endemic trachoma. The research provides up-to-date, population-based prevalence data of later complications of trachoma amongst a national sample of Indigenous adults.

Background: To report the prevalence of trachomatous trichiasis (TT) in Indigenous Australians aged 40 years and over.

Design: Population-based cross-sectional study

Participants: A total of 1738 (41\% male) Indigenous Australians aged 40 years or older, living among 30 randomly selected Australian sites, stratified by remoteness.

Methods: Anterior segment examination was performed and trachoma grading for the presence of $\mathrm{TT}$ and corneal opacification (CO) was conducted using the World Health Organization (WHO) simplified grading system.

Main outcome measure: Prevalence of $T T$

Results: A total of three (0.17\%) participants had $T \mathrm{~T}$ and there were no confirmed cases of trachomatous $\mathrm{CO}$ in the NEHS. All three participants with $\mathrm{TT}$ were female and aged 40 years or older. Although they had likely spent their childhoods in more remote 
areas, two of the three confirmed cases resided in an urban and outer regional area at the time of their examinations.

Conclusion and relevance: Our data is in line with on-going national trachoma surveillance reports that suggest the prevalence of late sequences of trachoma appear to be decreasing in Australia.

Keywords: trachoma, trichiasis, Indigenous health

This article is protected by copyright. All rights reserved. 


\section{NTRODUCTION}

Trachoma is caused by a bacterial (Chlamydia trachomatis) infection of the conjunctiva ${ }^{1}$ and remains the leading infectious cause of blindness worldwide. ${ }^{2}$ The disease has two phases, the initial "active stage" inflammatory response is usually seen in children. The late stage with scarring and structural changes in the eyelids causes trichiasis, entropion, corneal scarring and vision loss. ${ }^{3}$ Australia is the only developed country to still have pockets of endemic active trachoma (defined as prevalence $>5 \%)^{4}$ and known regional differences in the prevalence of trachoma exist, with rates being highest amongst very remote inland regions of New South Wales, the Northern Territory, South Australia and Western Australia. ${ }^{3,5}$

Supported by the World Health Organization (WHO), the Alliance for the Global Elimination of Blinding Trachoma by 2020 (GET 2020) promotes a four-component strategy to tackle endemic trachoma. ${ }^{6}$ Known as the SAFE strategy, this model includes; Surgery for trichiasis, Antibiotic treatment, Facial cleanliness and Environmental improvements. ${ }^{7}$ Since 2009, Australia has accelerated efforts towards fully implementing this strategy in order to meet the global targets by the year $2020 .^{9}$ The first national trachoma data for Australia came from the National Trachoma and Eye Health Program of the Royal Australian College of Ophthalmologists (NTEHP) in $1980 .{ }^{10}$ The overall national prevalence of active trachoma and $T \mathrm{~T}$ in this program were $17.6 \%$ and $6.0 \%$, with the prevalence of $T$ increasing to $34 \%$ in Central Australia. ${ }^{10}$ Despite significant improvements over the last 30 years, ${ }^{11}$ recent population-based studies have reported the prevalence of active trachoma is still common in children, particularly in very remote areas, and later complications of trachoma are still commonly observed in older Australians. ${ }^{5,12}$ The National Indigenous Eye Health Survey (NIEHS) (2008) reported an the overall national prevalence of active trachoma of 3.8\%, 
with endemic levels found in $50 \%$ of very remote communities. ${ }^{5}$ These rates are generally similar to reports from screening of I ndigenous school children conducted in the Northern Territory, South Australia and Western Australia in 2006 and 2007. 13,14 Furthermore, rates of later complications of trachoma in Indigenous adults aged 40 years and over have been recently documented, with the prevalence of $T T$ in the NIEHS ${ }^{5}$ and the Central Australian Ocular Health Study (CAOHS, 2010) $)^{12}$ reported to be $1.4 \%$ and $6.1 \%$, respectively. While both of these studies reported endemic levels of blinding trachoma in many very remote inland Indigenous communities, $T T$ was also observed in coastal and regional areas of Australia in the NIEHS. From this it is clear that despite notable improvements, trachoma remains a major health concern in many Indigenous Australian communities.

The purpose of this study is to report the prevalence of later complications of trachoma amongst Indigenous adults found in a national, population-based sample from the National Eye Health Survey (NEHS).

\section{METHODS}

\section{Study population}

The NEHS is a population-based survey that took place between March 2015 and April 2016 across thirty geographic areas in five Australian States and one Territory, stratified by remoteness. The sampling methodology of the NEHS has been described in detail elsewhere. ${ }^{15}$ In brief, a multi-stage random-cluster sampling methodology was utilised to identify thirty geographic areas across five Australian States and one Territory, stratified by remoteness, to obtain a representative sample of Indigenous Australians aged 40 years and older. In total, 12 major city, 6 inner regional, 6 outer regional, 4 
remote and 2 very remote sites were selected, corresponding to the approximate distribution of populations within each remoteness area.

Ethics approval was obtained from; the Royal Victorian Eye and Ear Hospital (RVEEH) Human Research Ethics Committee (HREC-14/1199H), the Aboriginal Health and Medical Research Council (AH\&MRC) of New South Wales (HREC-1079/15), the Menzies School of Health Research (HREC-2015-2360), the Aboriginal Health Council of Western Australia (AHCWA) (HREC-622) and the Aboriginal Health Council of South Australia (AHCSA) (HREC-04-15-604). Study procedures adhered to the tenets of the Declaration of Helsinki.

\section{Examination procedures}

Testing venues included culturally appropriate facilities that were easily accessed by community members including; community centres, schools, Aboriginal corporations, function centres, land councils, medical clinics, mobile clinics and town halls. All participants provided written informed consent. Information on participant demographics (date of birth, gender, level of education, country of birth, main language spoken at home), general health (e.g. diabetes and stroke), past ocular history and health service utilisation were obtained via self-report using an interviewer-administered questionnaire. Participants who identified as Indigenous were further classified into one of three categories that included; 1) Aboriginal, 2) Torres Strait Islander or 3) Aboriginal and Torres Strait Islander.

All ocular examinations were administered by ophthalmologists, optometrists, orthoptists or research assistants who were thoroughly trained under a standardised training protocol in all procedures and were under the supervision of an orthoptist or optometrist. Presenting distance visual acuity (VA) was assessed using a logMAR chart (Brien Holden Vision Institute, Australia) at $3 \mathrm{~m}$ in a well-lit room. Automated refraction was performed (Nidek Co., LTD, Japan) on participants whose VA improved to $\geq 6 / 12$ 
with pinhole in one or both eyes. Anterior segment assessment was conducted using a Keeler PSL hand-held slit lamp (Keeler Ophthalmic Instruments, UK) at 10x magnification. Participants with presenting distance VA of $<6 / 12$ in one or both eyes had anterior segment photographs taken using a non-mydriatic Diabetic Retinopathy Screening (DRS) camera (CenterVue SpA, Italy). Vision impairment and blindness were defined as having presenting visual acuity of $<6 / 12-6 / 60$ and $<6 / 60$, respectively. Trachoma grading for the presence of trachoma trichiasis $(T T)$ and corneal opacification (CO) was conducted using the WHO simplified grading system. ${ }^{16}$ In brief, $T$ was defined as the presence of one or more upper lid lashes touching the cornea, while CO was defined as the presence of a central corneal stromal opacity associated with other features of cicatricial trachoma. ${ }^{16}$ In this study, all participants underwent a slit-lamp assessment for the clinical signs of trachoma. Anterior segment photographs of participants with suspected trachoma were graded independently and in a masked manner by two ophthalmologists. In cases where anterior segment photo documentation was not available, the clinical grade as determined by the study optometrist was used.

Data analysis

All data was entered into a specialised online cloud-based database using tablet computers. The database was password protected, and each individual was allocated a unique identification code to maintain confidentiality. Following the collection of data for each participant, a checklist was completed by the examiner to ensure that all data were complete and valid. Participant demographic characteristics were summarised as the mean and standard deviation (SD) for normally distributed continuous data, and counts and percentages for categorical data.

\section{RESULTS}


A total of 2240 Indigenous residents were identified as eligible and 2,035 (90.8\%) agreed to participate. Of these, 1738 (77.6\%) Indigenous Australian adults ( 714 males and 1024 females) aged 40 to 92 years (mean age \pm standard deviation $=55.0 \pm 9.97$ years) were examined as part of the NEHS. Reasons for declining participation included; not interested ( $n=34,16.6 \%)$, no free time $(n=16,7.8 \%)$, previous bad research experience $(n=1,0.5 \%)$, a recent eye examination $(n=28,13.6 \%)$, refused to answer $(n=89,43.4 \%)$ and other $(n=37,18.0 \%)$. Compared to Indigenous male participants, Indigenous females had a significantly higher prevalence of self-reported diabetes (39.4\% vs. $33.9 \%, p=0.02)$ and a higher mean years of educational attainment (mean [SD] $=11.2$ [3.4] years vs. 10.7 [3.2], $p=\leq 0.001$ ) (Table 1). In total, 10 participants were suspected to have had sequelae of trachoma following clinical assessment by trained survey staff. Of these, anterior segment images were available for 7 of the 10 participants and $3(0.17 \%)$ participants were documented to have $\Pi$ via trachoma photograding. This may represent a rate in the total Indigenous population aged 40 years and over of $0.03 \%$. Of the 3 participants without anterior segment images, none had vision impairment or blindness and none were considered to have trachoma after further review of their medical and ocular histories. There were no confirmed cases of trachomatous $\mathrm{CO}$.

All three participants with $T T$ were female aged 40 years or older, with 1 residing in an urban region, 1 in an outer regional area and 1 in a remote area at the time of their examinations. The first case was an 83 year old Indigenous female, who had a history of stroke and diabetes (duration $=63$ years). Past ocular history revealed previous cataract surgery in both eyes with post-operative aphakia. Presenting visual acuity was 6/60 in each eye, with no significant improvement with pinhole. Grading of retinal images (two-field, 45 degree) revealed scattered dot hemorrhages of the right fundus 
and mild non-proliferative diabetic retinopathy and diabetic macular oedema of the left fundus. She had evidence of $T T$ in the right upper lid and senile entropion of the left lower lid with pannus in each eye. These findings were confirmed on anterior segment photos.

The second case was 40 year old Indigenous female who resided in an urban area. General health and past ocular history notes revealed self-reported diabetes (duration $=$ 15 years), trachoma and trichiasis surgery in both eyes 15 years ago. Presenting distance VA was 6/12pt in the right eye and 6/15 in the left eye, with no improvement in ether eye with pinhole. The fundus of each eye was unremarkable on retinal photography grading. She had $T T$ in the upper lids of both eyes and pannus in each eye, which were again confirmed on anterior segment photos.

The third case was a 48 year old Indigenous female residing in a remote area. Her general health, past ocular history and retinal grading results were unremarkable. Presenting distance VA was $6 / 15$ in the right eye and 6/12 in the left eye, with no improvement within either eye. There was $T \mathrm{~T}$ in the right upper lid with pannus in the right eye, which was confirmed in anterior segment photos.

\section{DISCUSSION}

In this study we report the prevalence of trachomatous trichiasis amongst Indigenous adults in the NEHS. Of all Indigenous participants aged 40 years and older, only $0.17 \%$ (3/1738) had $T \mathrm{~T}$ and none suffered $\mathrm{CO}$. This may represent a rate in the total population aged 40 years and over of $0.03 \%$.

Although trachoma is now confined to remote and very remote Indigenous communities, ${ }^{3}$ two of the three participants with $\Pi \mathrm{T}$ in the present study were tested in major city and regional areas. This finding is similar to the NIEHS that reported cases of 
$\pi$ in coastal and regional areas of Australia. ${ }^{5}$ Presumably, these participants will have spent their some of their childhood in remote or very remote endemic areas.

Additionally, all participants with $\Pi \pi$ in the current study were females. This is not consistent with most previous studies conducted in Australia but correlates with findings in other endemic countries ${ }^{17}$ where $T \mathrm{~T}$ and $\mathrm{CO}$ were approximately twice as common among women. One proposed reason for this gender predisposition relates to the traditional role of women in child raising, with a constant proximity to children resulting in an increased risk of repeated $C$. trachomatis infections. ${ }^{18}$ Despite this, due to the disproportionately higher number of females in our sample, this finding may simply be a reflection of the over-representation of females in the NEHS.

$\Pi T$ was confirmed in three cases and each had vision loss. The presence of bilateral aphakia was attributed as the main cause of vision loss in one case. In the absence of any significant fundus pathology or improvements on pinhole testing, $\Pi$ was suspected as a possible cause of vision loss in the remaining two cases. It should be noted, however, that we could not determine unequivocally that trachoma was the sole cause of vision loss in these cases.

The results of the current study can be readily compared to that of the $\mathrm{NIEHS}^{5}$ due to the employment of comparable methodologies and the similar examination rates achieved (NEHS $=78 \%$ vs. NIEHS $=79 \%$ ). The NIEHS reported TT amongst $1.4 \%$ of Indigenous adults aged 40 years and older. This is similar to the National Trachoma Surveillance Program and Reporting Unit (NTSRU) (2013) which reported TT among 1\% of participants across 183 at-risk communities in the Northern Territory, South Australia, Western Australia and New South Wales. ${ }^{11}$ The finding of a lower trachoma prevalence in the NEHS may indicate that initiatives targeting trachoma, ${ }^{19}$ coupled with clear improvements in housing water and sanitation, have had some success in lowering the overall prevalence of late complications of trachoma in Australia. Although 
the present study employed a similar sampling methodology to the NIEHS (random cluster, stratified by remoteness), vast differences in the regions of Australia that were sampled mean that these comparisons must be viewed with caution.

The prevalence of $T T$ in the present study is substantially lower than that reported in a similarly aged cohort in the CAOHS (6.1\%). ${ }^{12}$ This finding is likely to be explained by differences in areas sampled and the recruitment strategies between the two studies. That is, the CAOHS examined Indigenous individuals who attended an eye clinic held in remote and very remote inland areas where the prevalence of trachoma is highest. ${ }^{20}$ Strengths of the NEHS include the national scope, high response rates and the use of a standardised protocol to grade for $\mathrm{TT}$ and $\mathrm{CO}$. However, one key limitation must be considered. That is, a limited number of remote and very remote inland sites were sampled in the NEHS. Given I ndigenous communities in remote and very remote inland regions of Australia display the highest rates of trachoma, this is likely to have resulted in the low prevalence observed in the present study. Despite this, sampling in the NEHS was random and was designed to determine the national prevalence of all causes of vision impairment and blindness in Australia, rather than regional comparisons of the prevalence of trachoma.

In this national population-based survey, the prevalence of the late sequences of trachoma in Indigenous Australians aged 40 years and over was $0.17 \%$. This is considerably lower than that of previous population-based reports over the past decade, however comparisons are problematic due to differences in the regions sampled. ${ }^{5,12}$ While it is clear from on-going national surveillance that pockets of endemic levels of trachoma still exist, ${ }^{11}$ our data provides further support that the national prevalence of trachoma appears to be decreasing in Australia.

\section{Acknowledgements}


The Centre for Eye Research Australia (CERA) and Vision 2020 Australia wish to recognise the contributions of all the NEHS project steering committee members (Professor Hugh Taylor, Dr Peter van Wijngaarden, J ennifer Gersbeck, Dr J ason Agostino, Anna Morse, Sharon Bentley, Robyn Weinberg, Christine Black, Genevieve Quilty, Louis Young and Rhonda Stilling) and the core CERA research team who assisted with the survey field work (J oshua Foreman, Pei Ying Lee, Rosamond Gilden, Larissa Andersen, Benny Phanthakesone, Celestina Pham, Alison Schokman, Megan J ackson, Hiba Wehbe, J ohn Komser and Cayley Bush). Furthermore, we would like to acknowledge the overwhelming support from all collaborating Indigenous organisations who assisted with the implementation of the survey, and the Indigenous health workers and volunteers in each survey site who contributed to the field work.

The National Eye Health Survey was funded by the Department of Health of the Australian Government, and also received financial contributions from Novartis Australia and the Peggy and Leslie Cranbourne Foundation. In-kind support was received from our industry and sector partners, OPSM, Carl Zeiss, Designs for Vision, the Royal Flying Doctor Service, Optometry Australia and the Brien Holden Vision Institute. We would like to specifically acknowledge OPSM, who kindly donated sunglasses valued at \$130 for each study participant. The Centre for Eye Research Australia receives Operational Infrastructure Support from the Victorian Government. The Principal Investigator, Dr Mohamed Dirani, is supported by a NHMRC Career Development Fellowship (\#1090466). The PhD student, J oshua Foreman is supported by an Australian Postgraduate Award scholarship. 


\section{REFERENCES}

1. Taylor HR. Trachoma in Australia. Med J Aust 2001; 175: 371-2.

2. Pascolini D, Mariotti SP. Global estimates of visual impairment: 2010. Br J Ophthalmol 2012; 96: 614-8.

3. Warren JM, Birrell AL. Trachoma in remote Indigenous Australia: a review and public health perspective. Australian and New Zealand journal of public health 2016; 40 Suppl 1: S48-52.

4. Al HW. Eye health in Aboriginal and Torres Strait Islander People. In.Eye health in Aboriginal and Torres Strait Islander People, Vol. 2015, http://www.aihw.gov.au/WorkArea/DownloadAsset.aspx?id=10737418928:

AlHW, 2011.

5. Taylor HR, Fox SS, Xie J, Dunn RA, Arnold AL, Keeffe JE. The prevalence of trachoma in Australia: the National Indigenous Eye Health Survey. Med J Aust 2010; 192: 248-53.

6. WHO. Report of the 2 nd Global Scientific Meeting on Trachoma. In.Report of the 2nd Global Scientific Meeting on Trachoma,

http://www.who.int/blindness/2nd\%20GLOBAL\%20SCIENTIFIC\%20MEETING.pd

f: World Health Organisation, 2003.

7. Taylor HR, Burton MJ, Haddad D, West S, Wright H. Trachoma. Lancet 2014;

384: 2142-52.

8. WHO. Report of the Fifteenth Meeting of the WHO Alliance for the Elimination of

Blinding Trachoma by 2020. In.Report of the Fifteenth Meeting of the WHO

Alliance for the Elimination of Blinding Trachoma by 2020,

http://www.who.int/blindness/publications/GET15REPORT4.pdf: World Health

Organisation, 2011.

This article is protected by copyright. All rights reserved. 
9. CDNA. National guidelines for the public health management of trachoma. In.National guidelines for the public health management of trachoma, http://www.health.gov. au/internet/main/publishing.nsf/Content/D02F0C1C2AB90 509CA257C66001C089C/\$File/Trachoma-SoNG.pdf: Communicable Diseases Network Australia, 2011.

10. RACO. The national trachoma eye health program of the royal australian college of ophthalmologists. In.The national trachoma eye health program of the royal australian college of ophthalmologists, http://iehu.unimelb.edu.au/_data/assets/pdf file/0009/957411/Pt1.pdf: Royal Australian College of Ophthalmologists, 1980.

11. Cowling CS, Liu BC, Snelling TL, Ward JS, Kaldor JM, Wilson DP. Australian trachoma surveillance annual report, 2013. Communicable diseases intelligence quarterly report 2016; 40: E255-66.

12. Landers J, Henderson T, Craig J. Prevalence and associations of blinding trachoma in indigenous Australians within central Australia: the Central Australian Ocular Health Study. Clinical \& experimental ophthalmology 2010; 38: 398-404.

13. Tellis B, Keeffe J E, Taylor HR. Surveillance report for active trachoma, 2006: National Trachoma Surveillance and Reporting Unit. Communicable diseases intelligence quarterly report 2007; 31: 366-74.

14. Tellis B, Keeffe JE, Taylor HR. Trachoma surveillance annual report, 2007. A report by the National Trachoma Surveillance and Reporting Unit. Communicable diseases intelligence quarterly report 2008; 32: 388-99.

15. Foreman J, Keel S, Dunn R, van Wijngaarden P, Taylor HR, Dirani M. Sampling methodology and site selection in the National Eye Health Survey (NEHS): an 
Australian population-based prevalence study. Clinical \& experimental ophthalmology 2016.

16. Thylefors B, Dawson C, J ones B, West S, Taylor H. A simple system for the assessment of trachoma and its complications. Bull World Health Organ 1987; 65: 477-83.

17. Cromwell EA, Courtright P, King J D, Rotondo LA, Ngondi J, Emerson PM. The excess burden of trachomatous trichiasis in women: a systematic review and meta-analysis. Transactions of the Royal Society of Tropical Medicine and Hygiene 2009; 103: 985-92.

18. Congdon N, West S, Vitale S, Katala S, Mmbaga BB. Exposure to children and risk of active trachoma in Tanzanian women. Am J Epidemiol 1993; 137: 366-72.

19. Taylor H, Jatkar U, Anjou M. The roadmap to close the gap for vision. In.The roadmap to close the gap for vision, Vol. 2016, http://iehu.unimelb.edu.au/_data/assets/pdf_file/0007/1518802/RoadmapClose-Gap-for-Vision-Summary-Report-2015-September.pdf: Melbourne School of Population and Global Health, 2015.

20. Taylor HR. Trichiasis: out of mind, out of sight. The Australian journal of rural health 2009; 17: 171.

This article is protected by copyright. All rights reserved. 


\section{TABLE}

Table 1: Key demographics of Indigenous participants, stratified by gender

\begin{tabular}{lllll}
\hline Characteristic & $\begin{array}{l}\text { Total } \\
(\mathbf{n = 1 7 3 8 )}\end{array}$ & $\begin{array}{l}\text { Male } \\
(\mathbf{n}=\mathbf{7 1 4})\end{array}$ & $\begin{array}{l}\text { Female } \\
(\mathbf{n}=\mathbf{1 0 2 4})\end{array}$ & $\mathbf{p}^{*}$ \\
\hline Mean age (SD) & $55.0(10.0)$ & $55.1(9.8)$ & $54.9(10.1)$ & 0.69 \\
Educational attainment (years, SD) & $11.0(3.3)$ & $10.7(3.2)$ & $11.2(3.4)$ & $\leq 0.001$ \\
English spoken at home (n, \%) & $1671(96.1 \%)$ & $685(95.9)$ & $986(96.2)$ & 0.82 \\
Self-reported diabetes (n, \%) & $645(37.1 \%)$ & $242(33.9)$ & $403(39.4)$ & 0.02 \\
Self-reported stroke $(\mathrm{n}, \%)$ & $152(8.8 \%)$ & $72(10.1)$ & $80(7.8)$ & 0.13 \\
\hline
\end{tabular}

* $p$ values based on the chi-squared test for categorical variables or two independent samples t-test for continuous variables, comparing characteristics of I ndigenous male and female participants. Statistical significance was set as a $p$ value of $<0.05$ (twotailed). 


\section{University Library}

\section{- M M N E R VA A gateway to Melbourne's research publications}

Minerva Access is the Institutional Repository of The University of Melbourne

Author/s:

Dirani, M;Keel, S;Foreman, J;van Wijngaarden, P;Taylor, HR

Title:

Prevalence of trachomatous trichiasis in Australia: the National Eye Health Survey

Date:

2018-01-01

Citation:

Dirani, M., Keel, S., Foreman, J., van Wijngaarden, P. \& Taylor, H. R. (2018). Prevalence of trachomatous trichiasis in Australia: the National Eye Health Survey. CLINICAL AND EXPERIMENTAL OPHTHALMOLOGY, 46 (1), pp.13-17. https://doi.org/10.1111/ceo.13003.

Persistent Link:

http://hdl.handle.net/11343/293298 